\title{
Fluorescent false neurotransmitters
}

A fluorescent probe designed to incorporate a fluorophore into the structure of a neurotransmitter finds activity-dependent heterogeneity in dopamine release at individual synapses.

Electrophysiology has been a powerful tool for neuroscientists deciphering the rules for how the nervous system works at the cellular level. But the information it provides is limited. For example, the strength of synaptic connections between neurons that mediate signaling in neuronal networks is measured using recordings on postsynaptic cells, but this does not provide important information on changes in individual synapses between cells, which is considered the basis of learning and memory.

For Dave Sulzer at Columbia University this limitation has been an overriding frustration. "It's a really important question in neuroscience that people don't address because there hasn't been a means to do so," he says. But thanks to a collaboration between him and Dalibor Sames, also at Columbia University, this is no longer a limitation. They recently reported the creation of an optical probe they call a fluorescent false neurotransmitter (or FFN) and used it to look at activity changes in individual synapses during different levels of neuronal activity.

Sames and members of his lab are skilled at chemical synthesis and were recently looking for a neuroscientist to collaborate with on a fluorescent probe they had developed for an enzyme that metabolizes monoamine neurotransmitters. Sulzer thought the project would be interesting but remarked that what was really needed was a fluorescent dopamine to study neurotransmitter release at synapses. Fluorescent endocytic dyes have been used to study vesicle recycling, but according to Sulzer they haven't worked well for the dopamine system. Also, because the dyes bind to the vesicle membrane-unlike neurotransmitters, which are accumulated inside the vesicles - they do not really measure synaptic transmission.

The groups decided to create an FFN that targeted the neuronal vesicular monoamine transporter (VMAT) that transports neurotransmitters from the cytoplasm into synaptic vesicles. VMAT is less discriminating than cell-surface transporters, so it allowed them to look at a broad range of substrates. Former graduate student Niko Gubernator says: "I went into the library and searched through research papers to make lists of compounds that were substrates of VMAT." Using these lists, the Sames group started design work from scratch. Instead of attaching a fluorophore to a substrate, they merged the fluorophore and key structural features of VMAT substrates into single structures. Gubernator says: "Our biggest challenge was making them as small as possible while maintaining high fluorescence." Using this design approach, they synthesized seven candidate compounds.

Notably, the first time they tested one of the compounds (FFN511) in living slices of mouse brain by loading it for 30 minutes and then perfusing while imaging synapses during stimulation, it worked. They observed hundreds of presynaptic terminals simultaneously releasing the neurotransmitter. It turns out that all the compounds they had made covered a range of lipophilicities, and FFN511 was lipophilic enough for passive transport through the cell membrane into the cytoplasm so that VMAT could actively transport it into the vesicles where it became visible owing to the relatively high concentration in a tiny volume. Sames remarks: "We got lucky."

Their first results with the new probe are promising. "We've got the beginning of some rules," says Sulzer. "At basal firing rates - about 4 hertz - the activity of presynaptic terminals falls close to a normal distribution, but if we stimulate at 20 hertz, we see multiple populations of terminals, suggesting a mechanism for selecting individual synapses."

FFN511 seems well-suited to experiments in brain slices, and the researchers are developing a version for cultured neurons. It should also be possible to design FFNs to target other transporters and receptors. In all likelihood, FFN511 will soon be only one member of an expanding family of cellular probes.

\section{Daniel Evanko}

\section{RESEARCH PAPERS}

Gubernator, N.G. et al. Fluorescent false neurotransmitters visualize dopamine release from individual presynaptic terminals. Science 324, 1441-1444 (2009). 